Jolanta Hinc

Uniwersytet Gdański

\title{
ZUR FÖRDERUNG DER SPRACHBEWUSSTHEIT - VERGLEICHEN UND BESPRECHEN DER GRAMMATISCHEN STRUKTUREN
}

\section{Promoting language awareness - comparison and discussion of grammar structures}

\begin{abstract}
This paper elaborates on the notion of language awareness among multilingual students. In the first part the author presents the results of a research study whose objective was to assess if multilingual students are aware that the languages they speak and study (English and German) interact constantly and if they recognize the phenomenon of the positive and negative transfer. The subjects were students of Applied Linguistics at the universities of Bydgoszcz, Gdańsk and Poznań in Poland. The second part of the article is an attempt to connect theory and practice. It contains some suggestions how to improve multilingual foreign language teaching. A comparison and discussion of grammar structures aims to promote language awareness among students of foreign languages.
\end{abstract}

Keywords: multilingual students, language awareness, comparison of grammar structures

Słowa kluczowe: wielojęzyczność ucznia, świadomość językowa, porównywanie struktur gramatycznych języków

\section{Einleitung}

Der vorliegende Beitrag thematisiert die Förderung der Sprachbewusstheit multilingualer Lernender und Studierender, die durch Vergleichen und Besprechen der grammatischen Strukturen erfolgen soll. 
Der Beitrag setzt sich aus drei Teilen zusammen. Der erste Teil stellt die für die folgenden Überlegungen relevanten linguistischen und kognitiven Perspektiven der Mehrsprachigkeit dar. Außer der Erklärung des Transferbegriffs wird auf die Vergleichsdidaktik Bezug genommen, die zur Förderung der Sprachbewusstheit mehrsprachiger Lernender beitragen kann. Der zweite Abschnitt berichtet von den Ergebnissen einer Untersuchung, die 2013 unter Studierenden der Angewandten Linguistik an den Universitäten in Bydgoszcz, Gdańsk und Poznań durchgeführt wurde. Die Untersuchung zielte darauf ab, die Bewusstheit der Studierenden in Bezug auf den positiven und negativen Transfer zwischen den studierten Fremdsprachen nachzufragen. Der dritte Abschnitt des Beitrags ist praxisorientiert. Es wird davon ausgegangen, dass die Förderung der Sprachbewusstheit, die durch Vergleichen und Besprechen der grammatischen Strukturen und vor allem der für den negativen Transfer anfälligen Sprachbereiche der studierten Fremdsprachen erfolgen soll, ein Mittel sein kann, über die Parallelen und Differenzen in den Sprachsystemen zu reflektieren, auf den negativen Transfer aufmerksam zu machen und weiterhin den Interferenzfehlern vorzubeugen. Im Anschluss darauf wird eine didaktische Übung angeboten, die die Regularitäten zwischen den Sprachen im Bereich der Wortstellung explizit darstellt. Im vorliegenden Fall handelt es sich um die Sprachen Polnisch als Muttersprache, Englisch und Deutsch als Fremdsprachen.

\section{Linguistische und kognitive Perspektiven der Mehrsprachigkeit}

Das Forschungsinteresse für die vorliegende Untersuchung bezog sich auf die Tatsache, dass die früher gelernten (Fremd-)Sprachen interagieren, wenn eine neue Sprache erworben wird. Die Kenntnisse in früher erworbenen Sprachen üben einen positiven und einen negativen Einfluss auf das Erlernen einer weiteren Sprache aus. Der positive Transfer lässt die kommunikativ-pragmatische Kompetenz effizienter entfalten, wenn vorgängige Fremdsprachenkenntnisse und bisher angewandte Lernstrategien zu Hilfe genommen werden. Der negative Einfluss ist durch bestimmte Faktoren bedingt, vor allem durch die typologische Nähe der Sprachen (bei strukturell verwandten Sprachen steigt die Interferenzwahrscheinlichkeit), oder durch das Niveau der Sprachbeherrschung (die besser beherrschte Fremdsprache wird zur dominierenden Fremdsprache) (vgl. Cenoz 2001). Der negative Transfer äußert sich in der Fehlerproduktion, wenn Lernende gewisse Strukturen und Vokabeln entweder direkt oder indirekt aus der dominierenden Sprache übernehmen und auf die andere Sprache übertragen (vgl. Bouvy 2000, Groseva 1998, Hufeisen 1991). 
Kognitives Fremdsprachenlernen, das die Förderung der Sprachbewusstheit voraussetzt, bedeutet unter anderem auch einen vernünftigen, bewussten, sinnvollen und vor allem sprachvergleichenden Umgang mit allen Sprachen, die für die Lernenden relevant sind. Tönshoff (1991:161) differenziert zwischen konfrontierender und kontrastierender Bewusstmachung der Sprachphänomene. Erstere bedeutet den Einsatz kognitivierender Verfahren, die die Gegenüberstellung sprachlicher Elemente und Regularitäten und die Hervorhebung von Übereinstimmungen ermöglichen. Die zweite Art ist ein Teil der konfrontativen Bewusstmachung, bei der es sich um die Gegenüberstellung der Strukturen handelt.

Königs (1999:9) argumentiert, dass „der Fremdsprachenunterricht zumindest in einem vertretbaren Rahmen - auf kontrastiv angelegte Beschreibungen rekurrieren muss.“ Meißner (2003:31) bemerkt, dass „die Transferdidaktik eine Vergleichsdidaktik ist. Das Vergleichen verändert nicht nur das Wissen in der neuen Zielsprache, sondern auch in der bereits bekannten Sprache." Gozdawa-Gołębiowski (2005:103) betont, dass die Entwicklung der Interimsprache nur dann zustande kommen kann, wenn die Lernenden über grammatische Elemente ihrer Muttersprache zum Nachdenken angeregt werden, denn je mehr die Lernenden über ihre Sprache reflektieren, um so effektiver können sie die sprachlichen Phänomene der zu erlernenden Sprache erfassen. Der Einbezug der Muttersprache in den Sprachvergleich erweitert das Bezugsfeld für die zu erlernenden Fremdsprachen und trägt somit zur Intensivierung der Sprachaufmerksamkeit bei.

Das Vergleichen und Besprechen der grammatischen Strukturen stellt, wie oben angemerkt, einen Aspekt in der Entwicklung der Sprachbewusstheit multilingualer Lernender dar. Dieses Ziel formuliert Kleppin (2004:89) folgendermaßen: „Sprachbewusstheit in all ihren Ausprägungen könnte einerseits eine Hilfsfunktion für den Lernprozess übernehmen und andererseits als integraler Bestandteil von Mehrsprachigkeit gelten." Gnutzmann (1997:228) beschreibt Sprachbewusstheit allgemein als „Nachdenken über Sprache”. Nach Lutjeharms (1997:7) sei Sprachbewusstheit explizites Wissen über die Einsetzbarkeit, Anwendung und Übertragbarkeit, bzw. das Fehlen und die NichtÜbertragbarkeit von Sprachkenntnissen. Sprachvergleichende Aktivitäten dienen dazu, die Bewusstheit und Übertragbarkeit der Strukturen zu entwickeln.

Die Förderung der Sprachbewusstheit multilingualer Lernender soll auch die Problematik der interlingualen Interferenz umfassen. Dazu gehört die Bewusstmachung, dass

- paralleles Erwerben von mehreren Sprachen nicht additiv erfolgt, und dass alle Sprachen, einschließlich der Muttersprache, ständig interagieren (vgl. Grosjean 1982), 
- die Interaktion der Sprachen sich im positiven Transfer äußert, das heißt die sprachlichen Beziehungen zwischen der ersten und zweiten Fremdsprache erleichtern den Lernprozess, z.B. den Leseprozess durch den gemeinsamen Wortschatz,

- die Interaktion der Sprachen sich im negativen Transfer äußert, der das Erwerben der Sprachen erschwert, zu Interferenzerscheinungen führt und in der Produktion von Fehlern erscheint,

- die Interaktion der Sprachen sich im Kodewechsel äußert, das heißt im Umschalten zwischen verschiedenen Sprachsystemen.

- In der modernen vergleichenden Didaktik sollten für die Lernenden Möglichkeiten geschaffen werden über Sprachphänomene zu reflektieren, zu diskutieren und Parallelen und Differenzen in den Sprachsystemen zu entdecken. Das Ziel der Mehrsprachigkeitsdidaktik ist es somit, die Sprachbewusstheit der Lernenden zu fördern und sie dazu zu erziehen, die Anknüpfungsmöglichkeiten, die die Sprachverwandtschaft gewährt, zu nutzen, um den Lernprozess effizienter zu gestalten, das Verstehen neuer, sprachlicher Elemente zu erleichtern und Interferenzfehler erkennen und korrigieren zu können. In diesem Zusammenhang wird im Unterricht die konfrontative Bewusstmachung der Sprachphänomene angestrebt, deren Ziel auch sein soll, autonomes Lernen zu fördern.

\section{Untersuchung}

Die Untersuchung wurde im Frühjahr 2013 an drei polnischen Universitäten durchgeführt, an der Universität in Bydgoszcz, Gdańsk und Poznań.

Das Forschungsziel war die Bewusstheit multilingualer Studierender der Angewandten Linguistik, die Englisch und Deutsch studieren, in Bezug auf den positiven und negativen Transfer nachzufragen.

Das Forschungsinstrument war der Fragebogen mit sieben offenen Fragen (im Anhang). Die Fragen bezogen sich auf die folgenden Aspekte:

- die subjektive Einschätzung der dominierenden Fremdsprache,

- die Bewusstheit des positiven Transfers,

- die Bewusstheit des negativen Transfers, das heißt der Übertragung der Strukturen des Englischen auf das Deutsche und/oder des Deutschen auf das Englische,

- die Erwartung der Studierenden in Bezug auf die Berücksichtigung der Vergleichsdidaktik Englisch - Deutsch im Unterricht, 
- die aktuelle Situation im praktischen Englisch- und Deutschunterricht in Bezug auf die Vergleichsdidaktik Englisch - Deutsch.

Es wurden insgesamt 241 Studierende befragt, davon

- 30 Studierende des II. Studienjahres an der Universität in Bydgoszcz,

- 74 Studierende des I. und II. Studienjahres an der Universität in Gdańsk,

- 137 Studierende des I., II. und III. Studienjahres an der Universität in Poznań.

Die erhobenen Daten wurden quantitativ ausgewertet. Die Untersuchung erbrachte Folgendes:

- $\quad 86,6 \%$ der Befragten an der Universität in Bydgoszcz gaben Englisch als dominierende Sprache an, 72,9\% der Befragten an der Universität in Gdańsk gaben Englisch als dominierende Fremdsprache an, für 70,9\% der Befragten an der Universität in Poznań war Deutsch die dominierende Fremdsprache.

- $\quad 75,10 \%$ der Befragten erkennen den positiven Transfer. Hilfe bei der Einprägung neuer Wörter und bei der Antizipation der Bedeutung der Wörter waren die am häufigsten erteilten Antworten.

- $\quad 51,4 \%$ der Befragten sind sich des negativen Transfers bewusst.

- Den negativen Einfluss des Englischen auf das Deutsche bemerken 29,46\% der Probanden. In dieser Gruppe gaben 78,5\% der Befragten Englisch als dominierende Fremdsprache an. Die genannten Interferenzbereiche stellt die Grafik dar.

\section{Interferenzbereiche E $\rightarrow$ D}

Grammatik $\quad$ Wortschatz $\quad$ Rechtschreibung
ohne Kategorie Aussprache

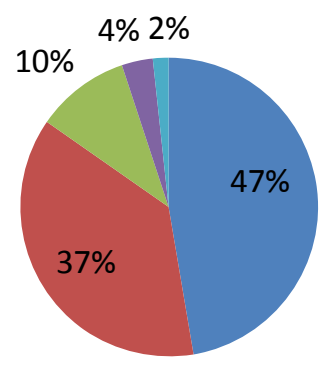

Abb. 1: Die Interferenzbereiche Englisch $\rightarrow$ Deutsch.

- Den negativen Einfluss des Deutschen auf das Englische bemerken $34,34 \%$ der Befragten. In dieser Gruppe gaben $60 \%$ der Befragten 
Deutsch als dominierende Fremdsprache an. Die anfälligsten Interferenzbereiche stellt die Grafik dar.

\section{Interferenzbereiche $\mathrm{D} \rightarrow \mathrm{E}$}

Grammatik Wortschatz $\quad$ Rechtschreibung
ohne Kategorie Aussprache

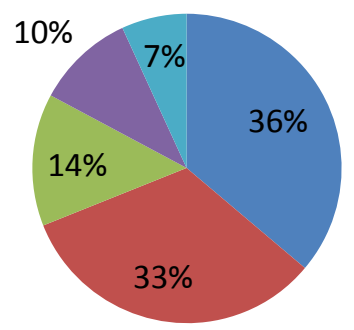

Abb. 2: Die Interferenzbereiche Deutsch $\rightarrow$ Englisch.

- 70,5\% der Befragten äußerten den Wunsch, die Sprachphänomene der studierten Fremdsprachen miteinander in Beziehung zu bringen, das heißt zu vergleichen und zu besprechen.

- $82,2 \%$ der Befragten gaben an, dass im praktischen Englisch- und Deutschunterricht auf die kontrastiv-konfrontative Analyse EnglischDeutsch kein Bezug genommen wird.

In Anbetracht der Tatsache, dass die Untersuchung 241 Studierende umfasste, können die Ergebnisse nicht als repräsentativ gelten. Das Ziel der Untersuchung war es, eine Tendenz aufzuzeigen und somit eine Grundlage für weitere Forschungen in diesem Bereich zu schaffen.

\section{Auswertung der Ergebnisse}

Die Untersuchung ergab, dass zwei Drittel der Befragten den positiven Transfer wahrnehmen. Die Kenntnis zweier Fremdsprachen erweist sich als hilfreich vor allem bei der Aufnahme neuer Vokabeln und bei der Antizipation der Bedeutung der Wörter. Gemeinsamkeiten auf der lexikalischen Ebene finden die Studierenden als besonders nützlich beim parallelen Erwerb des Englischen und Deutschen, das gemeinsame Sprachpotenzial wird somit aktiviert. Etwas über die Hälfte der Befragten nimmt den negativen Transfer wahr. In Anbe- 
tracht der Tatsache, dass ein Großteil der Probanden Englisch und Deutsch über mehrere Jahre lernt und dass in der Untersuchung Studierende der Angewandten Linguistik befragt wurden, scheint dieses Ergebnis erstaunlich niedrig zu sein. ${ }^{1}$ In Bezug auf die Bereiche, in denen die Befragten den negativen Transfer bemerken, nimmt die Grammatik die erste Stelle ein, gefolgt von Wortschatz und Rechtschreibung. Die letzte Stelle nimmt die Aussprache ein. Dieses Ergebnis betrifft sowohl die Übertragungen aus dem Englischen auf das Deutsche, als auch aus dem Deutschen auf das Englische. Im Bereich der Grammatik bemerken die Studierenden einen stärkeren negativen Einfluss des Englischen auf das Deutsche als des Deutschen auf das Englische. Dieses Ergebnis kann einerseits an der höheren Kompetenz der meisten Studierenden in Englisch liegen, andererseits an der Festigkeit der Regeln in den einzelnen Sprachbereichen des Englischen, etwa in der Wortstellung. Viele der Probanden nannten die Regeln der Anordnung der Elemente im Satz als das häufigste Beispiel des negativen Einflusses des Englischen auf das Deutsche.

Die meisten Befragten sehen es als wünschenswert, die grammatischen Strukturen des Englischen und Deutschen kontrastiv-konfrontativ zu behandeln und geben zu, dass sprachstrukturelle Vergleiche zwischen den beiden Sprachen in ihrem Unterricht nicht erfolgen. Es finden lediglich Seminare für die kontrastive Grammatik Englisch - Polnisch und Deutsch Polnisch statt. In Anbetracht der Sprachverwandtschaft des Englischen und Deutschen scheint hier ein wichtiges Prinzip der Fremdsprachenmethodik zu fehlen, nämlich die Aktivierung des deklarativen sprachlichen Wissens in der ersten Fremdsprache. Für die Optimierung und Ökonomisierung des Lernprozesses, vor allem aber für die Ausbildung sprachbewusster Fremdsprachenlehrer oder Übersetzer wäre angestrebt, bei den Studierenden die schon vorhandenen Sprachkenntnisse bewusst zu machen und zu erweitern, die Sprachverwandtschaft der Sprachen zu nutzen und zu entfalten, das heißt, die Strukturen zu vergleichen und zu besprechen und die Gemeinsamkeiten und Unterschiede festzustellen.

\section{Zur Förderung der Sprachbewusstheit - Vergleichen und Besprechen der Strukturen}

Die Untersuchung an der Angewandten Linguistik an den Universitäten in Bydgoszcz, Gdańsk und Poznań ergab, wie oben angemerkt, dass die meisten Studierenden es für unabdingbar halten, die Fremdsprachen, Englisch

\footnotetext{
${ }^{1}$ An der Universität Gdańsk ist das schriftliche Abitur in Englisch und Deutsch die Voraussetzung für die Aufnahme an der Angewandten Linguistik.
} 
und Deutsch, im Unterricht kontrastiv-konfrontativ zu betrachten. Daraus ergibt sich das Postulat, im Rahmen der pädagogischen Grammatik stärker auf dem Prinzip der vergleichenden Didaktik aufzubauen. Somit sollten im Unterricht Übungen angeboten werden, die die Regularitäten zwischen den Sprachen explizieren. Dies ist besonders wichtig, wenn zwei typologisch verwandte Sprachen parallel gelernt werden, wie es an der Angewandten Linguistik an den Universitäten in Bydgoszcz, Gdańsk und Poznań der Fall ist. Aber auch in den polnischen Grundschulen und Oberschulen sind Englisch und Deutsch die am häufigsten gewählten Fremdsprachen. Übungsbeispiele für den Vergleich der beiden Sprachen bieten Neuner und Hufeisen an (vgl. Neuner, Hufeisen 2003). Zwei wichtige Publikationen mit didaktischen Vorschlägen zum parallelen Lehren und Lernen des Englischen und Deutschen verfassten Wille, Wawrzyniak und Chłopek, Małgorzewicz (vgl. Chłopek, Małgorzewicz 2009; Wille, Wawrzyniak 2001).

Der Bereich der grammatischen Strukturen, die geeignetes Material für die sprachliche Kontrastierung und Besprechung im Unterricht darstellen, ist umfangreich. Zu den Strukturen zählen unter anderem: die Steigerung der Adjektive und Adverbien, die Passivbildung, Verben mit Präpositionalobjekt, die Struktur mit man oder die Modalwörter. Besonders begründet scheint die kontrastiv-konfrontative Analyse bei stark interferenzanfälligen Strukturen zu sein. Als das entsprechendste Beispiel erweist sich hier die Wortstellung. Im Bereich der Wortstellung ist die sog. Festigkeit der Wortstellungsregeln für die Interferenzwahrscheinlichkeit entscheidend. Die (Fremd-)Sprache, die die festeste Wortstellung aufweist, kann die Wortstellung der (Fremd-)Sprache, beinflussen, deren Wortstellungssystem im Vergleich dazu flexibler ist. So wies Ringbom (1987) empirisch nach, dass die englische Wortstellung die deutsche Wortstellung beeinflusst, wenn die Wortstellung der Muttersprache flexibler ist, als die Wortstellungssysteme des Englischen und des Deutschen. Laut Ringbom können die Lernenden nur die (Fremd-)Sprachen mit der festen Wortstellung vergleichen, die Regeln der flexiblen Wortstellung werden meistens nicht wahrgenommen und nur intuitiv angewandt. Aus der Sicht der Konstellation: Polnisch als Muttersprache, Englisch und Deutsch als Fremdsprachen weist das Polnische die flexibelste, Englisch die strengste Wortstellung auf. Übungen, die die Unterschiede in der Festigkeit der Worststellungssysteme explizieren würden, können die Lernenden auf den negativen Transfer in diesem Bereich aufmerksam machen.

Bei den vergleichenden Aktivitäten wird ferner angestrebt, auf die Strukturen der Muttersprache Bezug zu nehmen. Als Beispiel kann die von Gozdawa-Gołębiowski (2003) beschriebene Interface - Methode dienen. Sie setzt voraus, dass man das jeweilige sprachliche Phänomen in der 
Muttersprache diskutiert, bevor der neue Lehrstoff - die Struktur in der Fremdsprache - präsentiert wird. Auf diese Weise werden die Lernenden in die Problematik eingeführt, ihr deklaratives Wissen und ihre Sprachbewusstheit werden voraktiviert. So kann man vor der Einführung der Wortstellungsregeln in der Fremdsprache über Varianten der Wortstellung in der Muttersprache diskutieren. Dies soll die Lernenden in die Problematik der Anordnung der Elemente im Satz einführen und allgemeine, in vielen Sprachen auffindbare Prinzipien veranschaulichen, wie etwa die Stellung neuer Informationen nach bekannten oder die Möglichkeiten zur Hervorhebung besonders wichtiger Elemente im Satz (vgl. Paradowski 2006).

Als geeigneter Übungstyp bieten sich für den Sprachvergleich Übersetzungs- und Umstellungsübungen. Das unten stehende Beispiel ist ein Vorschlag für die Behandlung der Wortstellung, hier der Anordnung der Angaben. Übersetzen Sie bitte den folgenden Satz ins Englische und Deutsche.

\section{Byliśmy wczoraj $w$ restauracji.}

Antwort: Wir waren gestern im Restaurant. / We were in a restaurant yesterday. Stellen Sie in allen drei Sätzen die temporale Angabe um. Welche Variante klingt neutral?

Welche Variante ist sprachlich inkorrekt? Streichen Sie diese Variante durch. Byliśmy wczoraj $w$ restauracji./Wczoraj byliśmy $w$ restauracji./W restauracji byliśmy wczoraj.

Wir waren gestern im Restaurant./ Gestern waren wir im Restaurant./Wir waren im Restaurant gestern.

We were in a restaurant yesterday./ Yesterday we were in a restaurant./We were yesterday in a restaurant.

In welchem Satz ist die Zeit betont, in welchem der Ort? In welcher Stellung ist die Zeit bzw. der Ort eine neue Information?

Welche Gemeinsamkeiten und Unterschiede in Bezug auf die Stellung der Angaben im Satz entdecken Sie zwischen den Sprachen?

Fragen, die im Unterrichtsgespräch gestellt werden können, sollen die

Lernenden darauf aufmerksam machen, dass die Anordnung der Elemente im Satz auf die vorgenommene Sprechintention ankommt. Die Beispiele explizieren auch deutlich die Unterschiede zwischen den Sprachen. Vor allem aber werden die Lernenden bei Aktivitäten dieser Art zum Nachdenken angeregt, ihre intuitiven Gedanken und Wahrnehmungen werden verbalisiert und somit bewusst gemacht. In der Anfangsstufe kann das Unterrichtsgespräch in der polnischen Sprache geführt werden. Auf diese Weise werden die komplizierten metasprachlichen Formulierungen vermieden und die sprachlichen Barrieren abgebaut, so dass mehr Lernende zur Diskussion ermutigt werden können. 


\section{Abschließende Bemerkungen}

Durch bewussten Vergleich der Sprachphänomene und explizite Hervorhebung der Parallelen sowie der Differenzen zwischen den Sprachen ergibt sich die Möglichkeit, die Sprachbewusstheit der Lernenden zu fördern und sie auf den negativen Transfer vorzubereiten. Wann immer es sich also als hilfreich erweist, sollte gezielt auf die Gemeinsamkeiten und Unterschiede in den Sprachsystemen eingegangen werden. Dabei muss aber festgestellt werden, dass es sich bei dem Vergleich nicht um die Rückkehr zu der konfrontativen Analyse der 1960er und 1970er Jahre in ihrer ursprünglichen Form handelt. Das Prinzip des Vergleichens soll auch keineswegs mit der traditionell verstandenen Übersetzungsmethode gleichgesetzt werden. Im modernen Fremdsprachenunterricht dient der sprachstrukturelle Vergleich vielmehr der Förderung der Sprachbewusstheit. Sprachbewusste Lernende wissen die Strukturen je nach der Sprechintention und ihrer Adäquatheit für das angestrebte kommunikative Ziel einzusetzen, sie können die Sprachen vergleichen, sprachliche Analogien erkennen, einordnen und auswerten, den negativen Transfer bemerken und mit ihm umgehen. Übersetzungsübungen, die beim Vergleich unternommen werden können, hätten das Ziel, auf die Besonderheiten der einzelnen Sprachen explizit hinzuweisen.

Vergleichendes Vorgehen im Unterricht fordert aber von den Lernenden eine erhöhte Aktivität. Lernende, die Sprachphänomene vergleichend erarbeiten, über Regularitäten reflektieren und diskutieren, sind ideale Lernende. Darüber hinaus bemerkt Neuner (1999:16-18), dass die vergleichende Pädagogik allein noch keinen didaktischen Erfolg garantiert. Ein anderes oft erhobenes Gegenargument ist dieses, dass es an Lehrkräften mangelt, die über Sprachkenntnisse in zwei Fremdsprachen verfügen. Dies ist in Polen ein unbestreitbares Argument. Entgegenwirken kann man dieser Situation nur auf diese Weise, die Lernenden die führende Rolle beim Analysieren, Strukturieren und Erkennen von Gesetzmäßigkeiten spielen zu lassen. Somit lässt sich für den Unterricht als ein weiterer Lernzielbereich formulieren, die Fähigkeit zu entwickeln den eigenen Lernprozess zu reflektieren, das Sprachpotenzial zu nutzen und das Erlernen jeder weiteren Sprache vorzubereiten.

\section{BIBLIOGRAFIA}

Bouvy, Ch. 2000. „Towards the construction of a theory of cross-linguistic transfer” (w:) English in Europe: The acquisition of a third language. (red. J. Cenoz, U. Jessner). Clevedon: Multilingual Matters: 143-156. 
Cenoz, J. 2001. „The effect of linguistic distance, L2 Status and age on cross-linguistic influence in third language acquisition." (w:) Cross-linguistic influence in third language acquisition: psycholinguistic perspectives. (red. J. Cenoz, B. Hufeisen, U. Jessner). Clevedon: Multilingual Matters: 8-20.

Chłopek, Z., Małgorzewicz, A. 2009. Niebezpieczne podobieństwa angielsko-niemieckie. Gramatyka w ćwiczeniach. Warszawa: REA.

Gnutzmann, C. 1997. „Language Awareness. Geschichte, Grundlagen, Anwendungen“. Praxis des neusprachlichen Unterrichts 3: 227-236.

Gozdawa-Gołębiowski, R. 2003. Interlanguage formation. A study of the triggering mechanisms. Warszawa: Instytut Anglistyki UW.

Gozdawa-Gołębiowski, R. 2005. „Błąd językowy i gramatyka pedagogiczna”. Kwartalnik Pedagogiczny 1: 95-106.

Groseva, M. 1998. „Dient das L2-System als ein Fremdsprachenlernmodell?“ (w:) Tertiärsprachen. Theorien. Modelle. Methoden. (red. B. Hufeisen., B. Lindemann). Tübingen: Stauffenburg: 21-30.

Grosjean, F. 1982. Life with two languages. Cambridge: Harvard University Press.

Hufeisen, B. 1991. Englisch als erste und Deutsch als zweite Fremdsprache. Empirische Untersuchung zur fremdsprachlichen Interaktion. Frankfurt/Main: Peter Lang.

Jessner, U. 1997. „Towards a dynamic view of multilingualism“ (w:) Language choices: conditions, constraints, and consequences. (red. M. Pütz). Amsterdam: John Benjamins B.V.: 17-30.

Kleppin, K. 2004. „Mehrsprachigkeitsdidaktik = Tertiärsprachendidaktik? Zur Verantwortung jeglichen (Fremd-) Sprachenunterrichts für ein Konzept von Mehrsprachigkeit." (w:) Mehrsprachigkeit im Fokus. (red. K.R. Bausch, F.G. Königs, H.J. Krumm). Tübingen: Gunter Narr Verlag: 88-95.

Königs, F.G. 1999. „Mehrere Sprachen und Mehrsprachigkeit lernen! Fremdsprachenlerntheoretische Anmerkungen zur aktuellen Diskussion um Mehrsprachigkeit." (w:) Mehrsprachigkeit und Mehrkulturalität. Dokumentation des 18. Kongresses für Fremdsprachendidaktik, veranstaltet von der Deutschen Gesellschaft für Fremdsprachenforschung (red. K. Aguado, A. Hu). Berlin: Pädagogischer Zeitschriftenverlag: 1-17.

Lutjeharms, M. 1999. „Tertiärsprache und Sprachbewusstheit. Was Lernende über den Einfluss der ersten Fremdsprache denken." (w:) Fremdsprache Deutsch 1: 7-11.

Meißner, F.J. 2003. „Mehrsprachigkeitsdidaktik: Stand, Aufgaben, Entwicklungen im Überblick". Schriftenreihe Deutsch als Fremdsprache, II. Tagungsdokumentation, 2. Tagungsbeiträge und Arbeitsberichte zum Thema: Mehrsprachigkeit im Bereich Deutsch als Fremdsprache. (red. G. Neuner, U. Koithan). Kassel: Kassel University Press: 23-41.

Neuner G., „Deutsch nach Englisch. Übungen und Aufgaben für den Anfangsunterricht". Fremdsprache Deutsch 1: 15-21.

Neuner, G., Hufeisen B. et.al. 2003. Deutsch im Kontext anderer Sprachen. Tertiärsprachendidaktik: Deutsch nach Englisch. Fernstudieneinheit. Erprobungsfassung. Berlin und München: Langenscheidt. 
Paradowski, M.B. 2006. „Uczyć, aby nauczyć - rola języka ojczystego w gramatyce pedagogicznej i implikacje dla dydaktyki języków obcych." (w:) Komunikacja językowa w społeczeństwie informacyjnym. Nowe wyzwania dla dydaktyki języków obcych. (red. J. Krieger-Knieja, U. Paprocka-Piotrowska, Urszula). Lublin: Towarzystwo Naukowe Katolickiego Uniwersytetu Lubelskiego: 125-144.

Ringbom, H. 1987. The role of the first language in foreign language learning. Clevedon, Philadelphia: Multilingual Matters Ltd.

Tönshoff, W. 1991. Kognitivierende Verfahren im Fremdsprachenunterricht. Formen und Funktion. Hamburg: Dr. Kovac.

Wille, L., Wawrzyniak, Z. 2001. Deutsch nach Englisch im glottodidaktischen Gefüge. Ein Germanistenlehrbuch für Glottodidaktik und Methodik. Rzeszów: Wydawnictwo Wyższej Szkoły Pedagogicznej.

\section{Anhang}

Ankieta

1. Angielski czy Niemiecki? Który język w pani/pana subiektywnej ocenie zna Pani/Pan lepiej?

2. Czy zauważa Pani/Pan pozytywny wpływ języka angielskiego na naukę języka niemieckiego, lub odwrotnie, tzn. czy znajomość jednego języka ułatwia naukę drugiego? Jeśli tak, proszę podać przykłady.

3. Czy zauważa Pani/Pan negatywny wpływ języka angielskiego na naukę języka niemieckiego, lub odwrotnie, tzn. czy znajomość jednego języka utrudnia naukę drugiego? Jeśli tak, proszę podać przykłady.

4. Czy sądzi Pani/Pan, że na błędy popełniane przez Panią/Pana w języku angielskim może mieć wpływ Pani/Pana znajomość języka niemieckiego? Jeśli tak, proszę podać przykłady.

5. Czy sądzi Pani/Pan, że na błędy popełniane przez Panią/Pana w języku niemieckim może mieć wpływ Pani/Pana znajomość języka angielskiego? Jeśli tak, proszę podać przykłady.

6. Czy uważa Pani/Pan, że na zajęciach praktycznej nauki języka i gramatyki praktycznej powinno się porównywać struktury obu języków? Dlaczego?

7. Czy porównywane są struktury języka angielskiego i niemieckiego na zajęciach praktycznej nauki języka lub gramatyki praktycznej? 